\title{
Painful swelling of the knee and ankle: diagnosis and discussion
}

\author{
Rakesh Mohankumar • Haren Varia • Lipsita Patnaik • \\ Shabbir Susnerwala
}

Received: 10 October 2010 /Revised: 31 January 2011 / Accepted: 3 February 2011 /Published online: 22 February 2011

(C) ISS 2011

\section{Diagnosis}

Multi-focal epithelioid haemangioendothelioma of the bone and skin.

\section{Discussion}

Epithelioid haemangioendothelioma (EHE) is a rare, welldifferentiated vascular tumour of bone, accounting for only $0.5-1 \%$ of malignant primary bone tumours [1]. Weiss and Enzinger first described it in 1982 in soft tissues [2]. EHE is the most common epithelioid vascular neoplasm of bone.

EHE can present in locations other than bone, such as skin, soft tissue, liver, lungs, heart, intestine, nervous system, retroperitoneum and other body sites. However, it is most common in liver, soft tissues and bone [3].

The case presentation can be found at doi:10.1007/s00256-011-1127-1.

R. Mohankumar $\cdot$ H. Varia

Department of Radiology, Blackpool Victoria Hospital,

Whinney Heys Road,

Blackpool, Lancashire FY3 8NR, UK

L. Patnaik

Department of Histopathology, Blackpool Victoria Hospital,

Whinney Heys Road,

Blackpool, Lancashire FY3 8NR, UK

S. Susnerwala

Department of Clinical Oncology, Blackpool Victoria Hospital, Whinney Heys Road,

Blackpool, Lancashire FY3 8NR, UK

R. Mohankumar $(\bowtie)$

3, Ladybridge Avenue, Worsley,

Manchester M28 3BP, UK

e-mail: rakesh.mk@gmail.com
EHE in bone typically affects adults in the second or third decade, though all age groups can be affected. Sixty percent of EHE cases occur in the long bones with tibia (23\%), femur (18\%) and humerus $(13 \%)$ being the commonest sites [4]. Metaphysis and diaphysis are usually involved.

EHE presents as synchronous or metachronous disease in $20-50 \%$ of patients [4]. Multicentric presentation has been described in $50-62 \%$, with a predilection for bones of the lower extremities in one anatomic region [5]. Nearby or distant soft tissues and skin (Fig. 4) can be involved, and metastases to the lung have also been reported.

Conventional radiography of EHE shows an expansile, lytic and often poorly demarcated lesion. A coarse trabecular or honeycombing pattern is commonly seen, with a distinctive 'soap bubble' matrix. A well-defined sclerotic rim is seen in most lesions [6]. Calcification is rare, and periosteal reaction is not seen unless complicated by pathologic fracture [7]. Lesions with ill-defined borders are considered more aggressive (Fig. 1).

On MR imaging, the lesions are of intermediate signal intensity on T1-weighted images and high signal intensity on T2-weighted images. Homogenous enhancement is seen after administration of gadolinium. Flow voids due to vascular channels are not typically seen on MRI of EHE, and if present, should suggest an alternative diagnosis [4]. This could be due to the small size of the vascular channels [7] (Fig. 3).

Bone scintigraphy is often undertaken to identify the multicentric involvement of EHE. Increased uptake is seen at sites of involvement (Fig. 2). Doughnut-shaped lesions have been described at the site of involvement [8]. This appearance on bone scan can also be seen in benign or malignant lesions, but multiple lesions should raise the suspicion of a vascular lesion, such as haemangioendothelioma. 


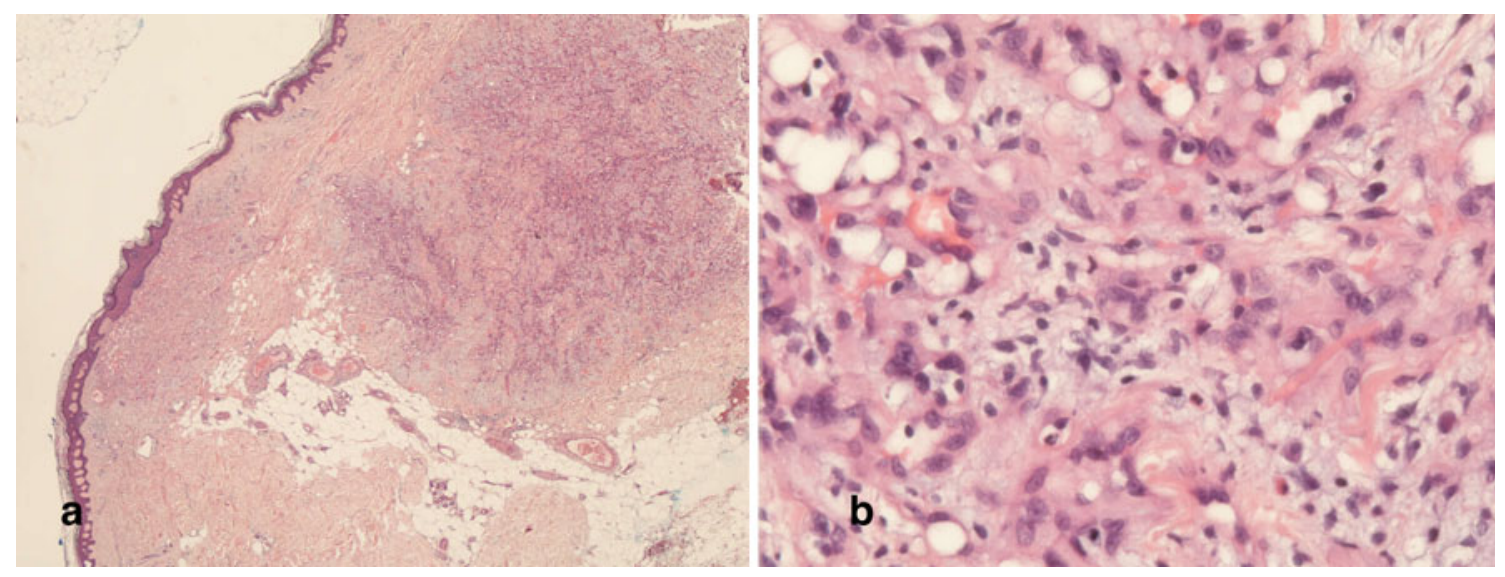

Fig. 5 a Low-power photomicrograph showing vascular tumour nodules (4×, H\&E). b High-power photomicrograph showing cords of tumour cells with intracytoplasmic vacuoles growing in a myxoid stroma $(40 \times, H \& E)$

The differential diagnosis of the imaging findings includes malignant lesions such as metastatic carcinoma and myeloma and benign lesions such as brown tumour of hyperparathyroidism [4-7].

Due to the non-specific imaging features, the final diagnosis is achieved on histopathology. Histologically, the tumour is composed of epithelial or histiocyte-like endothelial cells with abundant acidophilic and often vacuolated cytoplasm, large vesicular nucleus, modest atypia, scanty mitotic activity and inconspicuous or absent anastomosing channels (Fig. 5). Evidence of recent and old haemorrhage may be seen. A prominent fibro-myxoid stroma is characteristic of EHE. This differentiates it from the histological appearance of epithelioid haemangioma, which has an inflammatory stroma, and also does not show any atypia. Evidence of recent and old haemorrhage may be seen. EHE is generally categorised as a tumour of intermediate malignant potential. Visceral involvement is the most important criterion in predicting the prognosis of EHE [9].

The multifocal involvement affecting bones of one anatomical region should raise the suspicion of the pathology. Due to the propensity for multifocal and visceral involvement in EHE, evaluation with skeletal survey, bone scintigraphy and CT scan of the thorax and abdomen should be performed in every case. Due to the non-specific nature of the imaging findings, final diagnosis can only be attained by histology.
Disclosure The authors declare that there is no conflict of interest.

\section{References}

1. Grover SB, Mahato SK, Gupta P. Multicentric skeletal hemangioendothelioma with pulmonary metastasis. IJRI. 2004;14(4):4402.

2. Weiss SW, Enzinger FM. Epithelioid hemangioendothelioma: a vascular tumour often mistaken for a carcinoma. Cancer. 1982;50:970-81.

3. Turkmen C, Rozanes I. Hemangioendothelioma: whole body technetium $99 \mathrm{~m}$ red blood cell imaging - magnetic resonance imaging. In: Hayat MA. Cancer imaging (vol 2): instrumentation and applications. Burlington, MA: Academic Press; 2008. p. 281290.

4. Ignacio EA, Palmer KM, Mathur SC, Schwartz AM, Olan WJ. Resident's teaching files: epithelioid hemangioendothelioma of the lower extremity. Radiographics. 1999;19:531-7.

5. Bollinger BK, Laskin WB, Knight CB. Epithelioid hemangioendothelioma with multiple site involvement. Literature review and observations. Cancer. 1994;73:610-5.

6. Yoshida H, Watanabe M, Minamizaki T, Takasu N. Multicentric epithelioid hemangioendothelioma of the bone: histologic and radiographic features. Yonago Acta Med. 2000;43:87-92.

7. Larochelle O, Perigny M, Lagace R, Dion N, Giguere C. Epithelioid hemangioendothelioma of bone. Radiographics. 2006;26:265-70.

8. McNamara D, Beauregard GC, Lemieux RJ. Scintigraphic 'doughnut sign' on skeletal imaging due to a hemangioendothelioma of bone. J Nucl Med. 1993;34:297-300.

9. Kleer CG, Unni KK, McLeod RA. Epithelioid hemangioendothelioma of bone. Am J Surg Pathol. 1996;20:1301-11. 